\title{
AGE RELATED STRUCTURAL CHANGES IN HUMAN BASILAR ARTERY
}

\author{
Devika Gudiene, Ingrida Balnyte, Jolita Palubinskiene, \\ Angelija Valanciute
}

Lithuanian University of Health Sciences, Department of Histology and Embryology

\begin{abstract}
The basilar artery is the most important artery in the posterior cerebral circulation. In the cases of stroke due to acute basilar artery occlusion the mortality rate is significantly higher if compared to all the stroke cases. Arterial wall stiffening is characteristic of ageing arteries and in many investigations arterial wall stiffening is related to the loss of the elastic component in the arterial wall during ageing. In arterial stiffening the changes in the collagen content and the number of smooth muscle cells (SMC) may also play a role.

Basilar arteries were obtained within 24 hours postmortem from 89 human cadavers ( 44 male and 45 female). From the middle part of the basilar arteries histological slides were performed and area of collagen fibers, the number of smooth muscle cells and the thickness of the media were measured.

The morphometric analysis revealed the increase of collagen network area with the age. Analysing age-related changes in the number of SMC in male and female basilar artery media, we determined that in both genders the number of SMC in the media decreased. Changes in SMC number in both genders had a strong negative correlation with the age ( $\mathrm{r}$ $=-0.93$ in the male group and $\mathrm{r}=0.95$ in the female group, respectively). In the analysis of the media thickness in different gender and age groups, its thickening was determined. The correlation with the age was of medium strength both in the male $(\mathrm{r}=0.36)$ and female $(\mathrm{r}=0.4)$ group. In all the cases this correlation was statistically significant $(\mathrm{p}<0.05)$.

Our morphometric findings that revealed the increased collagen area together with the decrease of the SMC number might be responsible for
\end{abstract}


the stiffening of the basilar artery in aging and contribute to the development of atherosclerosis and arterial hypertension.

Key words: basilar artery, collagen fibers, media thickness, smooth muscle cells.

\section{INTRODUCTION}

Arterial tree aging is different in large elastic arteries and in distal muscular arteries [11]. It is commonly recognized that arterial aging is related to the arterial wall stiffening which is a physiological phenomenon assessed by the increased pulse wave velocity or augmentation index $[12,13]$. Experimental studies demonstrated that the effects of aging vary considerably along the cerebrovascular tree and the data obtained studying large arteries cannot be extrapolated to the smaller arteries of the circle of Willis [3].

The basilar artery is the most important artery in the posterior cerebral circulation. In the cases of stroke due to acute basilar artery occlusion the mortality rate is significantly higher if compared to all the stroke cases and the patients are about a decade younger [6]. Some authors consider that isolated basilar artery dissection is an underrecognized disease [14]. One of the methods to evaluate the arterial wall remodelling related to age is the morphometric investigation of the media. Scientific literature lacks information about the changes in the human basilar artery wall during aging. Fonck E. et al. [2] investigated another intracranial artery - the posterior cerebral artery and found considerable increase in the collagen content in the media with aging together with the decrease of smooth muscle cells (SMC) number.

Our aim was to investigate age-related changes in the basilar artery media by assessing the area of collagen fibers, the number of smooth muscle cells and media thickness.

\section{MATERIAL AND METHODS}

Basilar arteries were obtained within 24 hours postmortem from 89 human cadavers (44 male and 45 female, age from 20 to 84 years) at the Kaunas Department of M. Romeris University Institute of Forensic Medicine (the study was approved by the Kaunas Regional Ethics 
Committee for Biomedical Research, Protocol $\mathrm{Nr}$ BE-2-8). The investigation included the cases of violent death without obstructive atherosclerotic lesions and the history of cerebral blood vessels disease. All the investigated cases were distributed into 3 age groups: $<40$ years (young age group); 40-59 years (middle age group) and $\geq 60$ years (old age group).

Tissue samples were obtained from the middle portion of the basilar arteries and fixed in the 4\% neutral buffered formaldehyde. The tissue was dehydrated, embedded into paraffin and $5 \mu \mathrm{m}$ thick sections were performed. After that sections were stained with hematoxylin and eosin, lisamin fast red, picro-sirius red and acid orsein methods. Histological slides were observed with the optic microscope "Zeiss - standart 25" using the MC DX photocamera, 10 pictures from every case were investigated morphometrically using "Image-Pro Plus" v. 5.1 semiautomated image analysis system. The following quantitative parameters: the area (\%) of collagen fibers, the number of SMC and the thickness of the media were measured. The area of collagen fiber bundles was measured in percentage by calculating the total area of all the fibres and by dividing it by the area that we measured, magnifying $x$ 100. The morphometric analysis of collagen fibers was performed using the picro-sirius red method and counting of smooth muscle cells - the lisamin fast red method (Figure 1).

SMC were calculated quantitatively in the constant media areas of the same size. Also, in ten samples of each case ten calculations of the media thickness (in $\mu \mathrm{m}$ ) were made (100 calculations for each case). The slides stained with hematoxyline and eosine and acid orseine were used for the investigation of the histological structure of basilar arteries. For the statistical analysis SPSS 15.0 for Windows (SPSS Inc., Chicago, IL) was used ("Statistika 6.0" program). The initial morphometrical data distribution of each case testing the zero hypothesis refered to $\chi^{2}$ and Kolmogorov - Smirnov criterion was analysed. To compare histomorphometric parameters between two and more groups, we used the dispersive analysis using one and two factors method (ANOVA). Correlation between the analysed parameters was estimated by the correlation and regression analysis, $\mathrm{p} \leq 0,05$ was considered as statistically significant. 

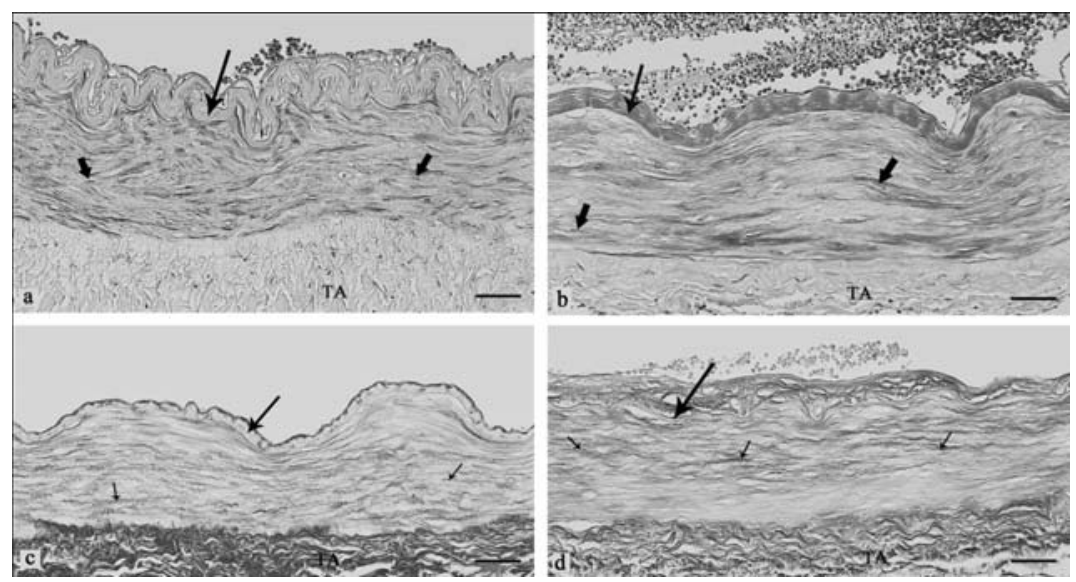

Figure 1. Histology of the basilar artery. a - male, age -27 years; $b-$ female, age 84 years, the diminished number of smooth muscle cells; c male, age -20 years; $d-$ male, age -65 years, the increased area of collagen fibers. TA - tunica adventitia, long arrows - the elastic membrane, short thick arrows $(a, b)$ - the nuclei of smooth muscle cells, short thin arrows (lisamin fast red), (c, d) - the collagen fibers are stained in red

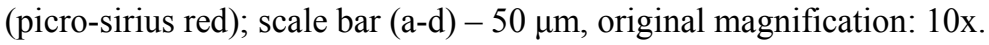

\section{RESULTS}

\section{Collagen network changes in the basilar artery in ageing}

The morphometric analysis revealed the increase of the collagen network area with the age. The area of collagen bundles was $3.4 \%$ higher in the male middle age group than in the young group $(\mathrm{p}<0.05)$. the difference between the old and middle age male groups the collagen area in media was $8.5 \%(\mathrm{p}<0.00001)$. There was $11.9 \%$ higher area of collagen network in the old age group than in the young age male $(\mathrm{p}<0.001)$ (Figure 2). The analysis of the female groups showed that there was significant difference of increase in the collagen network area in the basilar artery media between all the age groups $(\mathrm{p}<0.001)$. The area of collagen was $7.8 \%$ higher in the middle age group than in the young female and $5.6 \%$ lower than in the old age group. We found $13.4 \%$ higher collagen area percentage in the old female group than in the youngest one. 


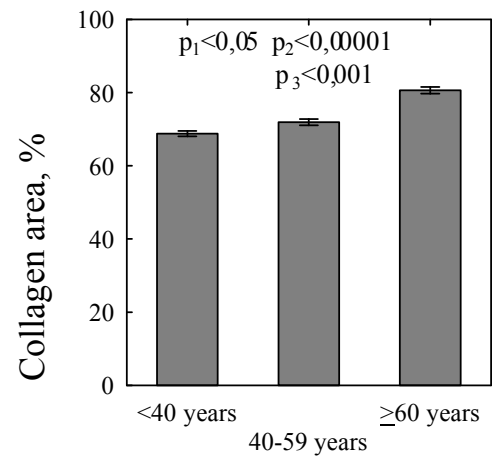

Male

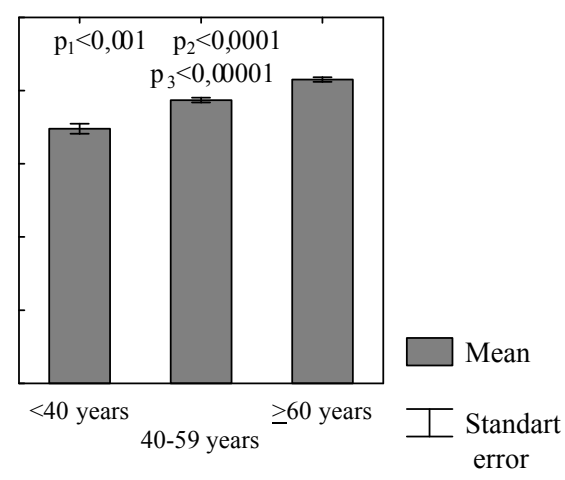

Female

Figure 2. Male and female collagen network area in the basilar artery media in all the age groups. $\mathrm{p}_{1}\left(<40\right.$ years and $40-59$ years); $\mathrm{p}_{2}(40-59$ years and $\geq 60$ years $) ; p_{3}(<40 \mathrm{~m}$ and $\geq 60$ years $)$.

Analyzing collagen network area of the same age male and female groups, we noticed that the collagen network area was higher in the female groups. This difference was statistically significant $(p<0.05)$ in the 40-59 age group.

\section{Age-related changes of the smooth muscle cells number}

Analysing age-related changes in the number of SMC in male and female basilar artery media, we determined that in both genders the number of SMC in the media decreased. Changes in SMC number in both genders had a strong negative correlation with the age $(r=0.93$ in the male group and $r=0.95$ in the female group).

We observed a statistically significant decrease in the SMC number in the old age group, where SMC decreased twice in comparison to the youngest group $(p<0.001)(53 \%$ of male, and $51 \%$ of female). The morphometric analysis of the data has shown that the number of SMC in the middle-aged group of male was lower by $26 \%$ than in the youngest group, but their number was higher by $36 \%$ than in the group of male older than 60 years $(\mathrm{p}<0.0001)$. The SMC number in the middle-aged female group was lower by $25 \%$ than in the group of female younger than 40 years and by $34.5 \%$ than the old age group of female $(p<0.001)$ (Figure 3). Such identical tendencies in the decrease of SMC in male 
and female of the same age demonstrate that the decrease in SMC in the basilar artery is similar in both male and female.

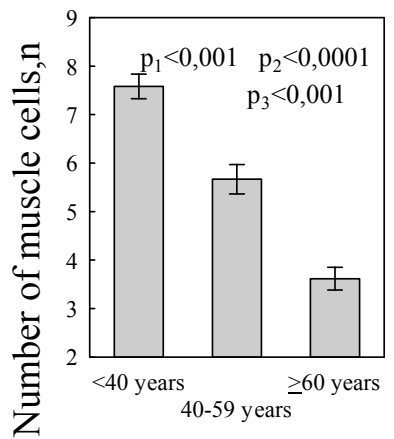

Male

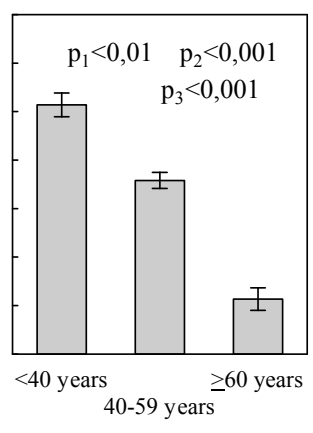

Female

Figure 3. Number of muscle cells in the basilar artery media. $\mathrm{p}_{1}(<40$ years and $40-59$ years); $\mathrm{p}_{2}$ (40-59 years and $\geq 60$ years); $\mathrm{p}_{3}(<40$ years and $\geq 60$ years).

The SMC number in the male basilar artery media was slightly bigger; this difference decreased with age. The number of SMC in the media of the male youngest group was higher by $10 \%$ than in the female group of the same age. It was higher by $8.3 \%$ in the middle-aged group of male and just by $5 \%$ in the old age group. This difference is statistically significant only between the youngest groups $(\mathrm{p}<0.05)$.

With the age the collagen content increases in the tunica media, while the SMC number decreases (Figure 4). In both gender groups the correlation between the collagen area and the SMC number in the arterial wall was statistically significant $(\mathrm{p}<0.05)$. In the male group a medium correlation was observed $(r=-0.5)$, whereas the correlation between the changes of the two parameters in the female group was strong $(\mathrm{r}=-0.7)$. Such results suggest that in the majority of cases the decreased SMC number is gradually replaced by collagen fibers, which causes thickening of the media. 


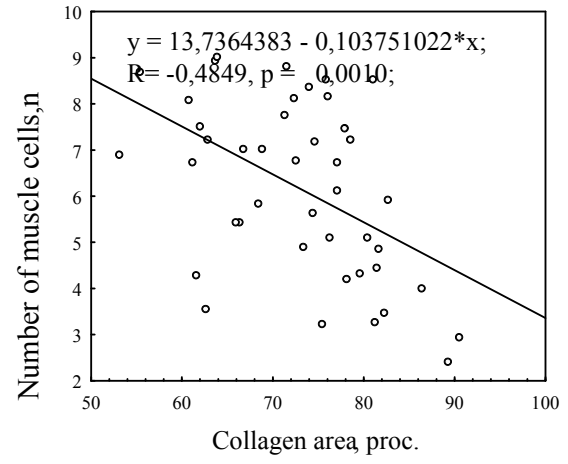

Male

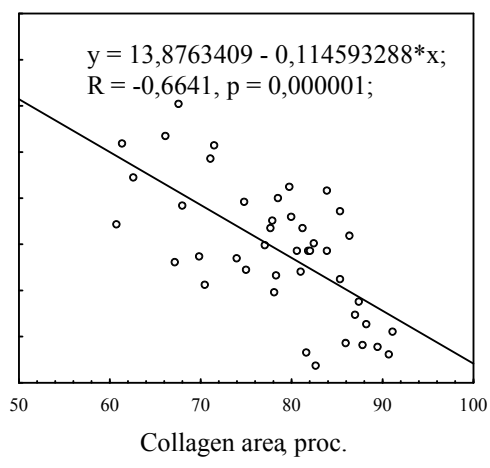

Female

Figure 4. The collagen area and the smooth muscle cells number correlation.

\section{Age-related changes of the media thickness}

In the analysis of the media thickness in different gender and age groups, its thickening with the age was determined. The correlation with the age was of medium strength both in the male $(\mathrm{r}=0.36)$ and female $(\mathrm{r}=0.4)$ group. In all the cases this correlation was statistically significant $(\mathrm{p}<0.05)$.

The media thickness in the male group younger than 40 years differed by $14.4 \%$ from the middle-aged group $(p<0.001)$ and by $22.5 \%$ from the oldest group $(\mathrm{p}<0.0001)$. In the male group over 60 , the media thickness was thicker by $7 \%$ than in the middle-aged group, and difference was statistically significant $(\mathrm{p}<0.0001)$.

The media thickness in female was smaller by $46.7 \%$ in the youngest group as compared to the middle-aged group, and smaller by $59 \%$ as compared to the oldest group. The media thickness was bigger by $8 \%$ in female over 60 than in the middle-aged group. In the female groups the differences in the media thickness were statistically significant $(\mathrm{p}<0.001)$. (Fig 5).

Similar percentages reflecting the changes of the media thickening in the oldest groups of different genders (in contrast to the younger groups) show that the changes in the media thickness differ between male and female more extensively in the younger age. Media thickening was sharper in the first two age groups of female than in those of male. After 
the age of 60 the rate of the changes almost equalled. The difference in the media thickness between the male younger than 40 and the older ones was just $14 \%$. The correlation between the changes in the media thickness and the collagen area was of medium strength in the male $(\mathrm{r}=0.38)$ and female $(\mathrm{r}=0.48)$ groups (Figure 6).

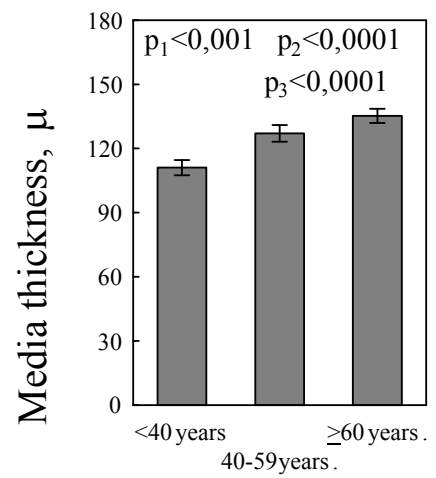

Male

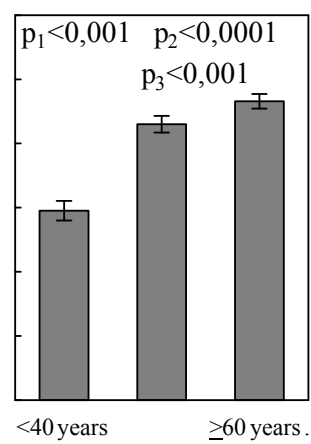

40-59years.

Fermale
Mean

I Standart error

Figure 5. Media thickness in male and female in all the age groups. $\mathrm{p}_{1}(<40$ years and $40-59$ years); $p_{2}$ ( $40-59$ years and $\geq 60$ years); $p_{3}$ ( $<40$ years and $\geq 60$ years).

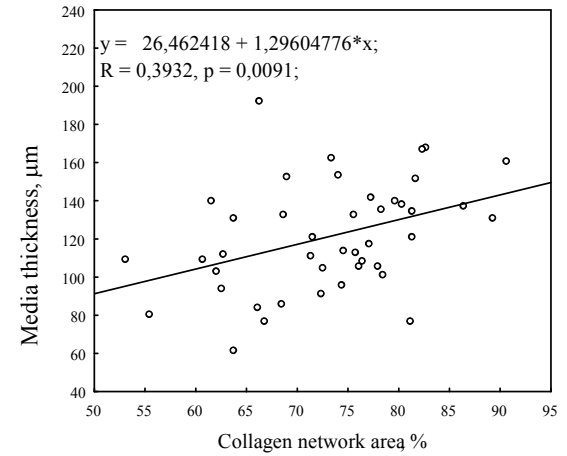

Male

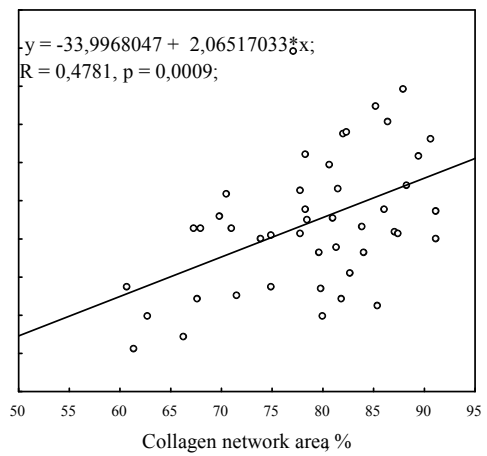

Female

Figure 6. Collagen fibers area and media thickness correlation in the basilar artery media. 
The correlation was statistically significant in both gender groups $(p<0.01)$. Such results show that the changes in the media thickness can be related to the increase of the collagen content in the basilar artery.

\section{DISCUSSION}

We determined that the human basilar artery collagen network area was increasing with the age. In our previous report we found that the perimeter of collagen bundles and their number were decreasing with age in both gender groups [4]. Changes and the regeneration of collagen network are going on constantly. Even in old age collagen fibers are fragmentating and forming again [5]. The area of the network increases, but the perimeter and the number of bundles decrease with the age. Similar age-related changes were noticed in the studies analyzing collagen network structure changes in other blood vessels and the heart $[1,7]$. While ageing collagen looses its toughness and the fibers start to stiffen and thicken [5]. With an increase of the amount of collagen, the elasticity of blood vessels decreases. The age-related structural evolution of arteries, with the predominance of collagen over elastin and reticulin, could be the reason of their fragility [7].

Our data also show the significant decrease of smooth muscle cells number in the media of the basilar artery during ageing. Statistical data have revealed that the decrease in the SMC number had a strong statistically significant negative correlation with the age $(p<0.05)$. Fonck E. et al. [2] investigated the age-related changes of the posterior cerebral artery and also found simultaneous diminishing of smooth cells number and the increase of the collagen content in the media of this artery.

When the thickness of the media of the basilar artery increases, the SMC number decreases. Similar data were obtained in some previous studies that analysed other cerebral arteries in relation to ageing [10]. We assume that the decrease in the SMC number does not have a direct influence on the media thickness. It is most likely that when the SMC number decreases, collagen fibers become thicker, which changes the properties and structure of the vascular wall.

In similar investigations of other arteries, researchers also relate the media thickening with the age, but concerning different age groups, they do not regularly obtain statistically significant data [8]. The correlation 
between the changes in the media thickness and the collagen area was of medium strength in the male and the female. This correlation was statistically significant in both gender groups $(p<0.01)$. Such results show that the changes in the media thickness can be related to the increase of the collagen content in the basilar artery. Our results support other researchers' conclusions [9].

Our morphometric findings that revealed the increased collagen area together with the decrease of SMC number might be responsible for the stiffening of the basilar artery in ageing and contribute to the development of atherosclerosis and arterial hypertension.

\section{REFERENCES}

1. Burkauskienè A. (2005) Age-related changes in the structure of myocardial collagen network of auricle of the right atrium in healthy persons and ishemic heart disease patients. Medicina, 41, 2,145-154.

2. Fonck E., Feigl G.G., Fasel J., Sage D., Unser M., Rüfenacht D.A., Stergiopulos N. (2009) Effect of aging on elastin functionality in human cerebral arteries. Stroke, 40, 7, 2552-6. Epub 2009 May 28.

3. González J.M., Briones A.M., Starcher B., Conde M.V., Somoza B., Daly C., Vila E., McGrath I., González M.C., Arribas S.M. (2005) Influence of elastin on rat small artery mechanical properties. Exp Physiol,90, 4, 463-8. Epub 2005 May 12.

4. Gudienè D., Valančiūtė A., Vèlavičius J. (2007) Collagen network changes in basilar artery in aging. Medicina, 43, 12, 964-970.

5. Hegedus K., Molnar P. (1989) Age-related changes in reticulin fibers and other connective tissues elements in the intima of the major intracranial arteries. Clin Neuropathol, 8, 2, 92-97.

6. Israeli-korn S.D., Schwammenthal Y., Yonash-Kimchi T., Bakon M., Tsabari R., Orion D., Bruk B., Molshatzki N., Merzeliak O., Chapman J., Tanne D. (2010) Ischemic stroke due to acute basilar artery occlusion: proportion and outcomes. Isr Med Assoc J, 12, 11, 671-5.

7. Johnson C.P., Baugh R., Wilson C.A., Burns J. (2001) Age related changes in the tunica media of the vertebral artery: implications for the assessment of vessels injured by trauma. J J Clin Pathol, 54, 2, 139-45.

8. Krus S., Turjman M.W., Flejka E. (2000) Comparative morphology of the hepatic and coronary artery walls. Part II. The relation between the internal elastic membrane, non-atherosclerotic intimal thickening and atherosclerosis. Med Sci Monit, 6, 2, 249-52. 
9. Marin J. (1995) Age-related changes in vascular responses: a review. Mech Ageing Dev, 79, 2-3, 71-114.

10. Masawa N., Yoshida Y., Yamada T., Joshita T., Sato S., Mihara B. (1994) Morphometry of structural preservation of tunica media in aged and hypertensive human intracerebral arteries. Stroke, 25, 1, 122-7.

11. Mitchell G.F., Parise H., Benjamin E.J., Larson M.G., Keyes M.J., Vita J.A., Vasan R.S., Levy D. (2004) Changes in arterial stiffness and wave reflection with advancing age in healthy men and women: the Framingham Heart Study.Hypertension, 43, 6, 1239-45.

12. Nürnberger J., Kribben A., Philipp T., Erbel R. (2007) Arterial compliance (stiffness) as a marker of subclinical atherosclerosis. Herz, 32, 5, 379-86. Review.

13. Lee H.Y., Oh B.H. (2010) Aging and arterial stiffness. Circ J, 74, 11, 2257-62. Epub 2010 Oct 15. Review.

14. Ruecker M., Furtner M., Knoflach M., Werner P., Gotwald T., Chemelli A., Zangerle A., Prantl B., Matosević B., Schmidauer C., Schmutzhard E., Willeit J., Kiechl S. (2010) Basilar artery dissection: series of 12 consecutive cases and review of the literature.Cerebrovasc Dis, 30, 3, 267-76. Epub 2010 Jul 24. Review.

\section{Adress for correspondence:}

Devika Gudiene

Lithuanian University of Health Sciences, the Department of Histology and Embryology Mickeviciaus 9, Kaunas LT 44307 Lithuania E-mail: balnyte@itc.kmu.lt 